Check for updates

Cite this: RSC Adv., 2018, 8, 20456

Received 8th May 2018

Accepted 23rd May 2018

DOI: $10.1039 / c 8 r a 03940 b$

rsc.li/rsc-advances

\title{
Effects of the inhibitor of glutamate decarboxylase on the development and GABA accumulation in germinating fava beans under hypoxia- $\mathrm{NaCl}$ stress
}

\begin{abstract}
Yongqi Yin, (D) Chao Cheng and Weiming Fang*
Glutamate decarboxylase (GAD) is the key enzyme in GABA shunt, which catalyzes the $\alpha$-decarboxylation of glutamate to produce GABA. A specific inhibitor for GAD is convenient to study the dynamic balances of GABA metabolism in plants. The inhibitor of GAD in germinated fava beans was screened, and its inhibitory effect on the growth and GABA accumulation in fava beans during germination under hypoxia$\mathrm{NaCl}$ stress was investigated. The inhibitory effect of aminoxyacetate for fava bean GAD was better than those of other chemicals, and it increased with the increase in concentration in vivo. After aminoxyacetate $(5 \mathrm{mM})$ application for 4 days during germination, the GAD activity in germinating fava beans was significantly inhibited by more than $90 \%$ in both organs. Meanwhile, the growth of fava bean sprouts was also slightly suppressed. Moreover, the GABA contents decreased by $43.9 \%$ and $81.5 \%$ in a 4 day-old cotyledon and embryo, respectively, under aminoxyacetate treatment compared with that in the control. In summary, these results showed that aminoxyacetate can serve as a specific inhibitor of GAD in plants. At least $43.9 \%$ and $81.5 \%$ of GABA in germinating fava beans under hypoxia- $\mathrm{NaCl}$ stress were synthesized via GABA shunt.
\end{abstract}

\section{Introduction}

$\Gamma$-Aminobutyric acid (GABA) is a ubiquitous four carbon, nonproteinaceous amino acid, which is one of the major inhibitory neurotransmitters in mammals. ${ }^{1}$ Recently, a huge amount of research has been done on the rapid accumulation of GABA levels in bean sprouts through seed germination under abiotic stresses, especially hypoxia and salt stress, ${ }^{2-5}$ as GABA can prevent certain forms of cancer and reduce the risk of cardiovascular diseases. ${ }^{6,7}$ In higher plants, the conserved metabolic pathways known as the GABA shunt and polyamine (PA) degradation pathways have been well studied. GABA is synthesized from alpha-decarboxylation of glutamate (Glu) catalyzed by glutamate decarboxylase (GAD, EC 4.1.1.15) in the cytosol; this process is called the GABA shunt. ${ }^{8}$ Meanwhile, polyamine (PA) oxidation is catalyzed by diamine oxidase (DAO, EC 1.4.3.6) and polyamine oxidase (PAO, EC 1.5.3.11) to form $\gamma$-amino butyraldehyde and then, $\gamma$-aminobutyraldehyde is converted by aminoaldehyde dehydrogenase (AMADH, EC 1.2.1.19) to produce GABA. ${ }^{9}$

Mechanisms have been put forward for the production of GABA, the dynamic balances of GABA shunt and PA degradation pathway in plants. At present, the effect of the PA degradation pathway on GABA accumulation was investigated by inhibiting

College of Food Science and Technology, Yangzhou University, Yangzhou, Jiangsu 210095, People's Republic of China. E-mail: wmfang@yzu.edu.cn; Fax: +86-51489786551; Tel: +86-514-89786551
DAO activity via aminoguanidine (AG).,10 The reduction in GABA formation was considered to be due to PA degradation pathway after DAO activity was inhibited. It has been reported that about $30 \%$ of GABA accumulation is due to PA degradation in germinated fava beans under non-stress ${ }^{\mathbf{1 1}}$ and hypoxia stress. ${ }^{2}$ However, there is no specific inhibitor for GAD to investigate the contribution of GABA shunt on GABA accumulation in plant tissues under stress, and the contribution rate of the GABA shunt to GABA accumulation is not clear, although the GABA shunt has been studied since it was first reported more than fifty years back. ${ }^{\mathbf{1 2}}$ Furthermore, AG is a specific inhibitor of DAO rather than PAO and AMADH. More and more research has demonstrated that AG cannot inhibit DAO activity completely ${ }^{\mathbf{1 0 , 1 1 , 1 3}}$ let alone PAO and AMADH, which are also key enzymes responsible for GABA synthesis in the polyamine degradation pathway. ${ }^{9}$ A specific inhibitor for GAD, as the only known rate-limiting synthase in GABA shunt, is convenient to study the accurate contribution ratio of the GABA shunt for GABA formation. Besides, further studies on the GABA shunt will undoubtedly provide a new understanding of the mechanism of GABA accumulation.

GAD has been identified and purified in various higher plants, and its enzymatic properties have been studied in detail. ${ }^{\text {14-16 }}$ Considerable inhibitory potentials of certain reagents have been confirmed in vitro, particularly the inhibitory effects on purified plant GAD. ${ }^{\mathbf{1 4}}$ These in vitro studies indicated that sulfhydryl compound and aminoxyacetate can inhibit the activity of purified GAD. ${ }^{\mathbf{1 4 1} 17}$ To the best of our 
knowledge, very few studies have reported the effects of these reagents on the physiological functions of plants, especially the metabolic process of GABA. The aim of this study is to confirm the in vivo inhibitory effect of four selected inhibitors. Meanwhile, in case of the specific inhibitor applications, the changes in the growth and the contribution of GABA shunt in GABA accumulation of fava beans during germination under hypoxia$\mathrm{NaCl}$ stress are investigated.

\section{Experimental}

\subsection{Materials and reagents}

The fava bean seeds (Qi Bean 2) were purchased from Jiangsu Academy of Agricultural Sciences (Nanjing, China). Aminoxyacetate, GABA, dimethylaminoazobenzene sulfonyl chloride and Glu were purchased from Sigma Chemical Co. (St Louis, MO, USA).

\subsection{Material treatment and experimental design}

Dried fava bean seeds were surface-sterilized by soaking in $1 \%$ $(\mathrm{v} / \mathrm{v})$ sodium hypochlorite for $15 \mathrm{~min}$; then, they were washed and steeped in deionized water for $8 \mathrm{~h}$ at $30^{\circ} \mathrm{C}$. The soaked seeds were put into a culturing pallet $(25 \mathrm{~cm} \times 20 \mathrm{~cm} \times 5 \mathrm{~cm})$ and incubated in the dark at $30{ }^{\circ} \mathrm{C}$ for $48 \mathrm{~h}$. The seeds were sprayed with deionized water every $8 \mathrm{~h}$. Then, the pretreated fava beans were placed in sprout pots $(6 \mathrm{~cm}$ diameter $\times 9 \mathrm{~cm}$ height) and germinated in a dark incubator at $30{ }^{\circ} \mathrm{C}$ for 4 days under hypoxia-NaCl stress ( $\mathrm{pH}$ 3.5, $10 \mathrm{mM}$ citrate buffer, $60 \mathrm{mM}$ $\mathrm{NaCl}$, dissolved oxygen concentration $5.5 \mathrm{mg} \mathrm{L}^{-1}$ ). The condition of hypoxia-NaCl treatment was chosen because of the high GABA content in germinated fava beans, as indicated in our previous study. ${ }^{18}$ Meanwhile, the culture solution contained 0.0 , 2.5, 5.0, 7.5 and $10.0 \mathrm{mM}$ of $\beta$-mercaptoethanol, dithiothreitol, L-cysteine and aminoxyacetate, respectively. Three independent biological experiments were performed. During the germination, the culture solution was replaced at $8 \mathrm{~h}$ intervals. The sprouts were collected, rinsed, frozen in liquid nitrogen, and stored at $-20{ }^{\circ} \mathrm{C}$ until analyses.

Moreover, the pretreated fava bean seeds were germinated under hypoxia-NaCl stress as described above, and the culture solution contained $5.0 \mathrm{mM}$ of aminoxyacetate. The control sprouts were germinated under the culture solution without inhibitors. The seeds were germinated for $0 \mathrm{~d}, 1 \mathrm{~d}, 2 \mathrm{~d}, 3 \mathrm{~d}$ and 4 d. Then, the sprouts were also collected, rinsed, frozen and stored for analyses.

\subsection{Measurements of GABA and Glu contents}

GABA and Glu were extracted from germinated fava beans and purified according to the procedure described by Bai et al. ${ }^{19}$ The residue was dissolved in $2 \mathrm{~mL}$ of $1 \mathrm{M}$ sodium bicarbonate $(\mathrm{pH}$ 9.0) and centrifuged at $6000 \mathrm{~g}$ for $10 \mathrm{~min}$. GABA and Glu contents were determined using the Agilent 1200 Series HPLC system with a ZORBAX Eclipse AAA reverse phase column (150 mm × $4.6 \mathrm{~mm}, 3.5 \mu \mathrm{m}$; Agilent, Palo Alto, Calif., USA), as described by Yang et $a .^{2}$

\subsection{GAD activity assay}

GAD activity was determined according to the method described by Bai et $a .^{19}$ The GABA content in the reaction solution was analyzed by the standard method. One unit of GAD activity was defined as the release of $1 \mu \mathrm{M}$ of GABA produced from glutamate per $60 \mathrm{~min}$ at $40{ }^{\circ} \mathrm{C}$. The GAD activity of plant tissues was defined as units of GAD activity of $1 \mathrm{~g}$ DW.

\subsection{Measurements of sprout length and fresh weight}

Sprout length was measured directly using a vernier caliper. Thirty sprouts were set as a sampling group for each measurement. For determination of fresh weight (FW) and dry weight (DW), 30 soybeans were weighed, followed by ovendrying at $80{ }^{\circ} \mathrm{C}$ for $48 \mathrm{~h}$ and then, the average weight was calculated.

\subsection{Statistical analysis}

All results were expressed as mean values \pm standard deviations of three replicates independent of the germinated fava beans. The data obtained were subjected to analysis of variance (ANOVA), and the mean differences were compared with Tukey's test; a $p$ value at 0.05 was considered significant.

\section{Results and discussion}

\subsection{Selection of GAD inhibitor}

The present study illustrated the in vivo inhibitory effects of sulfhydryl compounds and aminoxyacetate on germinated fava bean GAD activity in various concentrations (Table 1). GAD activities in cotyledon and embryo of germinating fava beans showed a dose-dependent decrease with an increase in the concentrations of the selected inhibitors $0.0 \mathrm{mM}$ onwards. In comparison with the results for the control $(0.0$ $\mathrm{mM}$ ), the GAD activities in both organs were significantly inhibited, as was observed at $0.25 \mathrm{mM}$ of all the inhibitors. After treatment with $\beta$-mercaptoethanol, dithiothreitol and $\mathrm{L}^{-}$ cysteine, the GAD activity in the embryo was found to be higher than that in cotyledon at the same concentration, whereas the results were different under aminoxyacetate treatment. GAD activity in both organs significantly decreased up to $7.5 \mathrm{mM}$ of $\beta$-mercaptoethanol, dithiothreitol and L-cysteine and then remained constant following any further increase in their levels (Table 1). The fava beans were germinated for 4 days under $5.0 \mathrm{mM}$ of aminoxyacetate treatment, after which it was found that the GAD activities in embryo were $53.6 \%, 17.3 \%$ and $21.5 \%$ for the $\beta$-mercaptoethanol, dithiothreitol and L-cysteine treatments, respectively, at the same concentration. Moreover, the GAD activity was not detected in cotyledon and embryo of the germinated fava beans treated with $10.0 \mathrm{mmol} \mathrm{L}^{-1}$ aminoxyacetate. These results suggested that aminoxyacetate maybe the appropriate inhibitor for GAD activity during fava bean germination. 
Table 1 Effect of sulfhydryl compound and aminoxyacetate on GAD activity ${ }^{a}$

\begin{tabular}{|c|c|c|c|c|c|c|}
\hline \multirow[b]{2}{*}{ Inhibitors } & \multirow[b]{2}{*}{ Organs } & \multicolumn{5}{|c|}{ Concentration $\left(\mathrm{mmol} \mathrm{L}^{-1}\right)$} \\
\hline & & 0.0 & 2.5 & 5.0 & 7.5 & 10.0 \\
\hline & Embryo & $18.88 \pm 0.64 a$ & $4.50 \pm 0.10 \mathrm{~b}$ & $3.45 \pm 0.15 c$ & $2.29 \pm 0.03 d$ & $1.98 \pm 0.04 d$ \\
\hline \multirow[t]{2}{*}{ Dithiothreitol } & Cotyledon & $15.37 \pm 0.55 \mathrm{a}$ & $9.33 \pm 0.49 b$ & $8.81 \pm 0.60 \mathrm{~b}$ & $5.90 \pm 0.19 c$ & $5.01 \pm 0.68 \mathrm{c}$ \\
\hline & Embryo & $18.88 \pm 0.64 \mathrm{a}$ & $11.19 \pm 0.58 b$ & $10.67 \pm 0.20 \mathrm{~b}$ & $7.28 \pm 0.29 c$ & $6.93 \pm 0.69 c$ \\
\hline \multirow[t]{2}{*}{ Aminoxyacetate } & Cotyledon & $15.37 \pm 0.55 \mathrm{a}$ & $2.85 \pm 0.20 \mathrm{~b}$ & $2.38 \pm 0.02 b$ & $0.78 \pm 0.02 \mathrm{c}$ & Not detected \\
\hline & Embryo & $18.88 \pm 0.64 a$ & $3.12 \pm 0.16 b$ & $1.85 \pm 0.03 c$ & $0.79 \pm 0.02 \mathrm{~d}$ & Not detected \\
\hline
\end{tabular}

${ }^{a}$ Lower case letters reflect the significance of differences in GAD activity among chemical concentrations $(p<0.05)$.

\subsection{Changes in GABA and Glu contents and sprout lengths after inhibition of GAD activity}

The GABA content significantly decreased $(p<0.05)$ in cotyledon and embryo of germinated fava beans exposed to aminoxyacetate (Table 2). After treatment with $5.0 \mathrm{mM}$ aminoxyacetate, the GABA contents in cotyledon and embryo decreased by $66.03 \%$ and $90.32 \%$, respectively, in comparison with the results for the untreated control. The Glu content showed a different response, that is, its content increased first at low concentrations of aminoxyacetate and then, it started markedly declining at higher concentrations of aminoxyacetate (Table 2). The Glu contents in cotyledon and embryo of 4 d germinated fava beans increased 1.56- and 1.53-fold, respectively, when compared with those of the control. The sprout length of the germinating fava bean, understandably, was progressively suppressed with the increase in aminoxyacetate concentration, whereas there was no significant difference found when the concentration of aminoxyacetate was above $5.0 \mathrm{mM}$ (Table 2). The above-mentioned results suggested that the optimal inhibition concentration of aminoxyacetate in germinated fava beans was $5.0 \mathrm{mM}$.

\subsection{Growth performance of germinated fava bean}

To evaluate the effect of aminoxyacetate on the germinated fava bean, its sprout length and fresh weight were measured during germination (Fig. 1). Compared with that of the control, the sprout length of the fava bean decreased during germination in a aminoxyacetate-treated sprout (Fig. 1A). After germinating for
4 days under aminoxyacetate treatment, the sprout length was $65.7 \%$ of that of the control. Similarly, the inhibitory effect of aminoxyacetate was also observed on the fresh weight (Fig. 1B). The fresh weight increased significantly $(p<0.05)$ with germination time under treatment; however, after 4 days, aminoxyacetate addition resulted in a decrease by $12.9 \%$ compared with the results for the control. The growth of germinating fava beans was inhibited under aminoxyacetate treatment during germination.

\subsection{Changes in GAD activity in germinating fava bean}

Under aminoxyacetate treatment, the GAD activities in cotyledon and embryo during germination are shown in Fig. 2 . Under the normal hypoxia-NaCl stress condition, the GAD activity increased first and then decreased during germination, showing peak values at $3 \mathrm{~d}$ and $2 \mathrm{~d}$ of germination in cotyledon and embryo, respectively. As expected, aminoxyacetate treatment led to a continuous decrease in GAD activity, which was always maintained at a lower level during germination in both organs (Fig. 2). These facts proved that aminoxyacetate is a specific inhibitor of GAD and can be selected appropriately for the research regarding the mechanism and dynamic balances of GABA in higher plants.

\subsection{Changes of GABA and Glu contents in germinating fava bean}

GABA contents in cotyledon and embryo both showed a continual and significant enhancement under normal

Table 2 Effect of aminoxyacetate on GABA content, Glu content and sprout length in hypoxia- $\mathrm{NaCl}$ stressed fava bean ${ }^{a}$

\begin{tabular}{|c|c|c|c|c|c|}
\hline \multirow{2}{*}{$\begin{array}{l}\text { Aminoxyacetate } \\
\left(\mathrm{mmol} \mathrm{L}^{-1}\right)\end{array}$} & \multicolumn{2}{|c|}{ GABA content $\left(\mathrm{mg} \mathrm{g}^{-1} \mathrm{DW}\right)$} & \multicolumn{2}{|c|}{ Glu content $\left(\mathrm{mg} \mathrm{g}^{-1} \mathrm{DW}\right)$} & \multirow[t]{2}{*}{ Sprout length $(\mathrm{mm})$} \\
\hline & Cotyledon & Embryo & Cotyledon & Embryo & \\
\hline 2.5 & $1.31 \pm 0.10^{\mathrm{b}}$ & $1.91 \pm 0.23^{\mathrm{b}}$ & $104.52 \pm 11.26^{\mathrm{a}}$ & $99.42 \pm 6.76^{\mathrm{b}}$ & $7.8 \pm 0.2^{\mathrm{b}}$ \\
\hline 5.0 & $0.96 \pm 0.06^{\mathrm{c}}$ & $1.25 \pm 0.12^{\mathrm{c}}$ & $106.93 \pm 10.21^{\mathrm{a}}$ & $145.87 \pm 19.58^{\mathrm{a}}$ & $6.9 \pm 0.1^{\mathrm{c}}$ \\
\hline 7.5 & $0.53 \pm 0.08^{\mathrm{d}}$ & $0.67 \pm 0.03^{\mathrm{d}}$ & $57.91 \pm 6.05 b^{c}$ & $67.84 \pm 5.87^{\mathrm{c}}$ & $6.6 \pm 0.1^{c}$ \\
\hline
\end{tabular}

${ }^{a}$ Lower case letters reflect the significance of differences in GABA content, Glu content and sprout length among chemical concentrations $(p<0.05)$. 

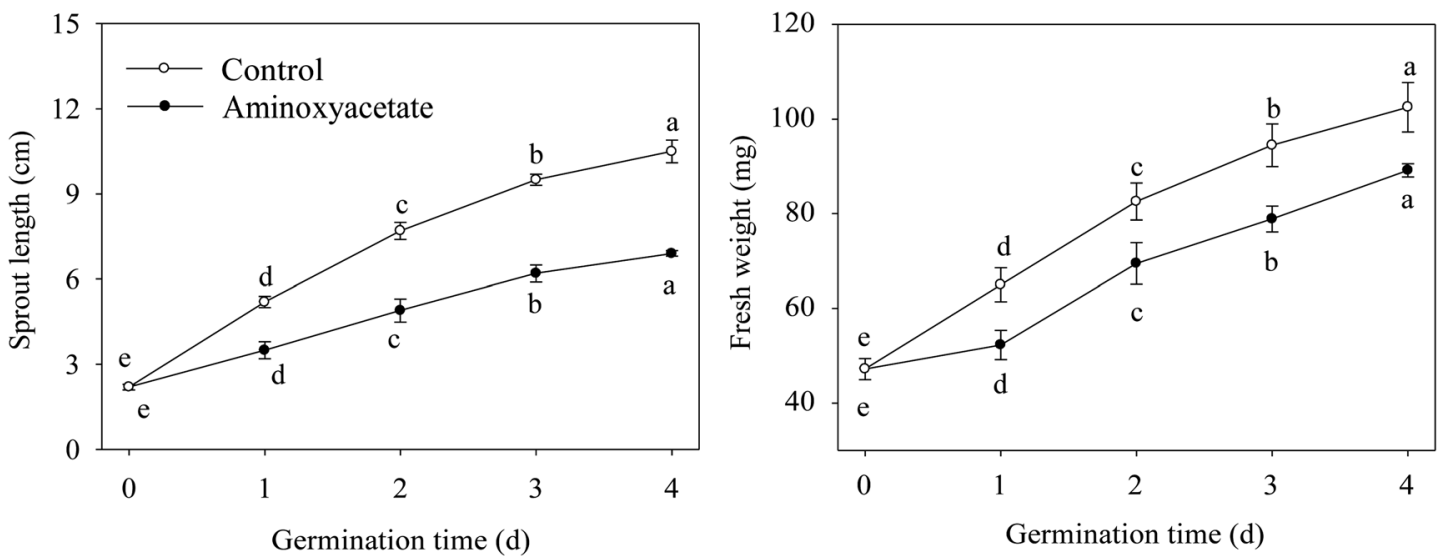

Fig. 1 Effect of aminoxyacetate on sprout length and fresh weight of germinated fava bean. Lower case letters reflect the significance of differences in sprout length and fresh weight during germinating under their respective treatments $(p<0.05)$.

hypoxia-NaCl stress during germination, and the GABA content was higher in the embryo when compared with that in the cotyledon (Fig. 3A and B). Under aminoxyacetate treatment, the GABA content gradually increased as the germination time was prolonged. Simultaneously, the aminoxyacetate treatment resulted in a noticeable decrease in GABA contents by $43.9 \%$ and $81.5 \%$ in the cotyledon and embryo, respectively, of $4 \mathrm{~d}$ germinated fava beans when compared with the results of the untreated control. In the early stage of germination, the Glu contents in fava beans continued to increase under both treatments, but the contents exhibited decrease in later stages. The Glu contents in cotyledon and embryo under aminoxyacetate treatment remained higher than those of untreated control during the whole germination process (Fig. 3C).

\subsection{Discussion}

In plants, GABA is synthesized via GABA shunt and polyamine degradation pathway. Abiotic stress can significantly increase the GAD and DAO activities and promote GABA accumulation during seed germination..$^{\mathbf{8} 9}$ The primary biosynthesis of GABA in plants is carried out by the action of cytosolic GAD. ${ }^{8}$ Studies on GAD are required for a better understanding of the metabolic mechanism of GABA in plants. There have been numerous studies that have investigated the regulation and activity of GAD in different plants; ${ }^{20-22}$ also, the enzymatic properties of plant GAD have been studied in detail. ${ }^{15-17}$ Plant GAD generally contains a C-terminal region known as the $\mathrm{Ca}^{2+} /$ calmodulin (CaM)-binding domain..$^{23}$ Hence, the study indicated that GAD activity can be inhibited by sulphydryl reagents, aminoxyacetate, calcium binding agents, 3-mercaptopropionate, etc. ${ }^{24-26}$ However, there is very less information about the effects of these inhibitors on the physiological and metabolic processes of GABA in plants. It was evident from our study that $\beta$-mercaptoethanol, dithiothreitol and L-cysteine showed dosedependent inhibitory control abilities in fava bean GAD during germination. Consistent with previous in vitro results, ${ }^{2,25}$ the suppression effect of aminoxyacetate on GAD activity in germinating fava beans under hypoxia- $\mathrm{NaCl}$ stress was still better than those of other chemical inhibitors in the present study.

Not surprisingly, $5.0 \mathrm{mmol} \mathrm{\textrm {L } ^ { - 1 }}$ of aminoxyacetate not only inhibited GAD activity but also suppressed the growth of fava bean sprouts during germination, since the GABA shunt has been proven to play an important role in plant carbon and nitrogen partitioning and development. ${ }^{\mathbf{8} 20}$ Moreover, Barbosa
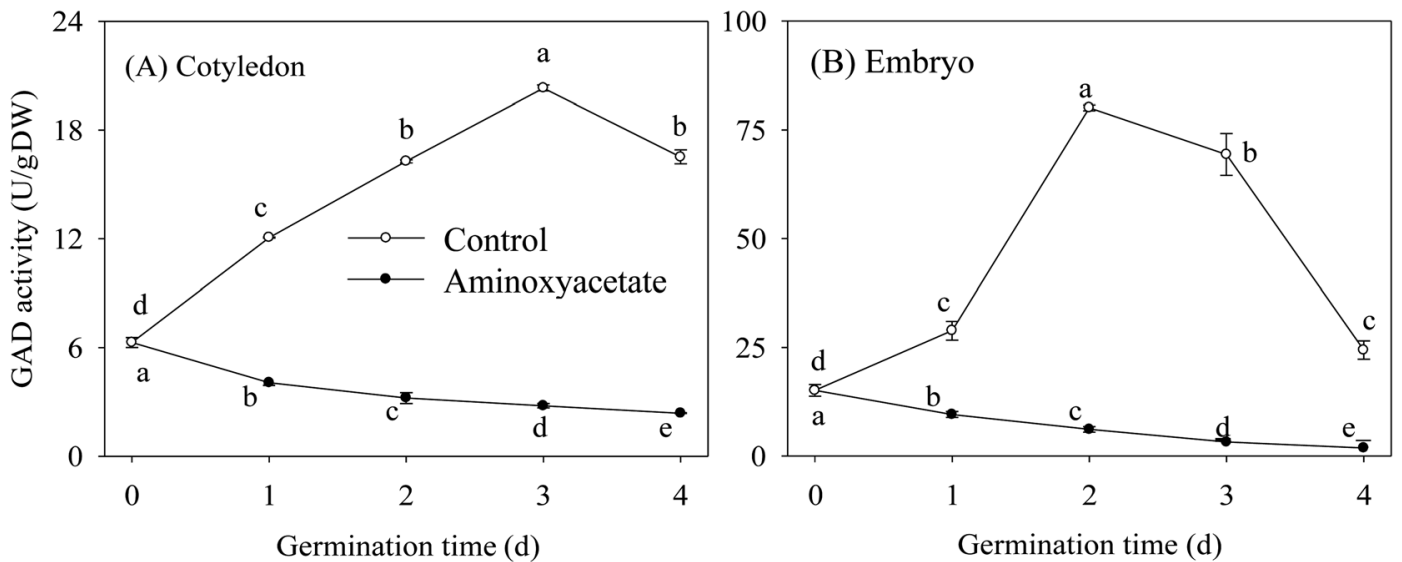

Fig. 2 Effect of aminoxyacetate on GAD activity of germinated fava bean. Lower case letters reflect the significance of differences in GAD activity during germinating under their respective treatments $(p<0.05)$. 

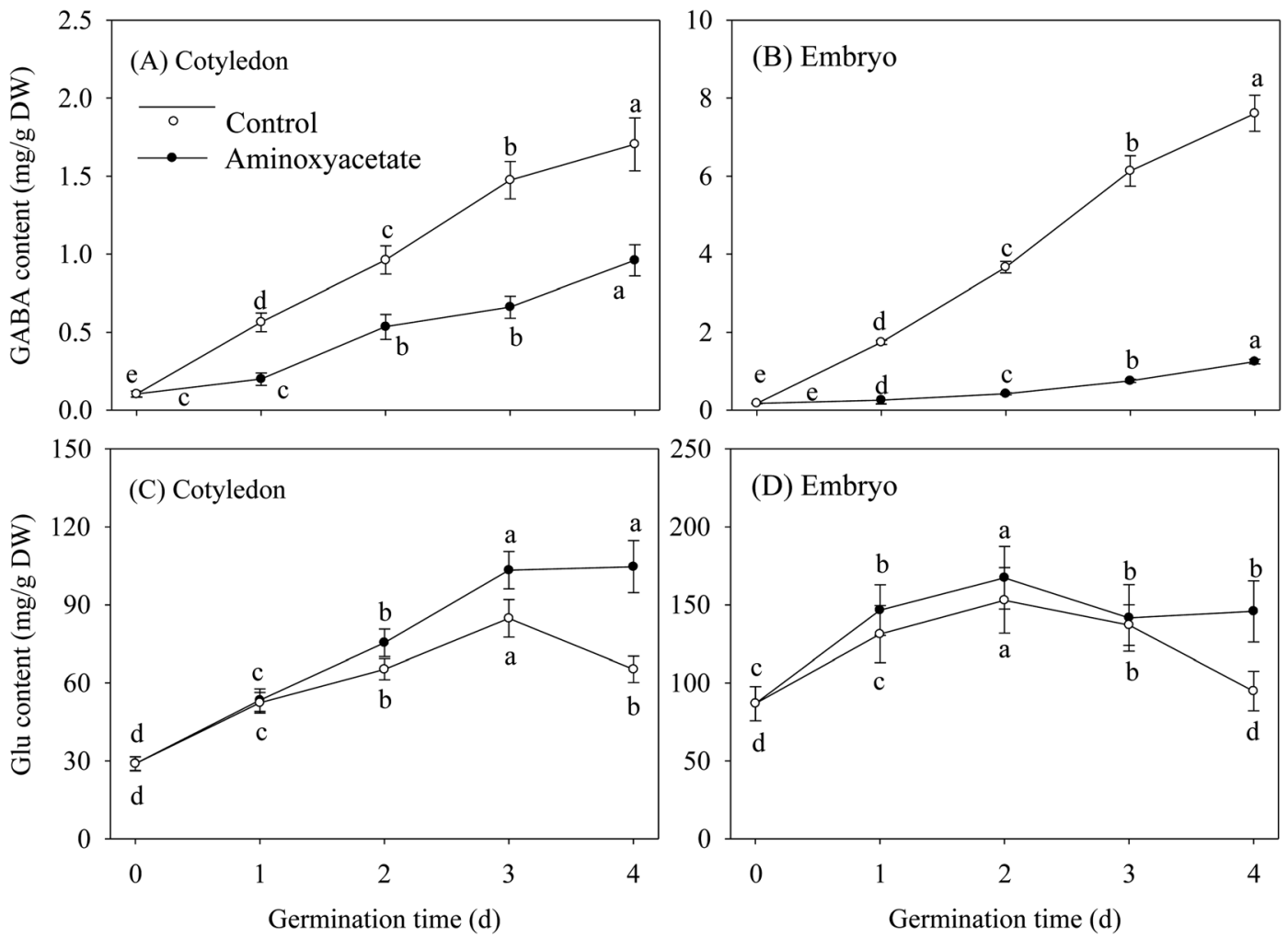

Fig. 3 Effect of aminoxyacetate on GABA and Glu contents of germinated fava bean. Lower case letters reflect the significance of differences in GABA and Glu contents during germinating under their respective treatments $(p<0.05)$.

et al. suggested that the plant GAD plays a critical role in the regulation of $\mathrm{pH}$, mineral acquisition and nitrogen uptake. ${ }^{27}$ GAD activity may also have a relationship with the earlier diversification and evolution of vascular plants. ${ }^{28}$ In the present research, aminoxyacetate inhibited $90.1 \%$ and $94.5 \%$ of GAD activities in cotyledon and embryo, respectively, of $4 \mathrm{~d}$ germinated fava beans. Subsequently, the GABA contents in the cotyledon and embryo decreased by $43.9 \%$ and $81.5 \%$, respectively, compared with those of the control. It can be concluded that in cotyledon and embryo of the germinating fava beans under hypoxia- $\mathrm{NaCl}$ stress, the GABA shunt provided at least $43.9 \%$ and $81.5 \%$ of GABA formation as GAD activity was not inhibited completely. Previous researchers have found that between $30 \%$ and $40 \%$ of GABA accumulation is contributed by the polyamine degradation pathway in germinated fava beans under hypoxia stress by the inhibition of DAO activity via AG. ${ }^{11}$ Meanwhile, AG inhibited $39 \%$ and $24.5 \%$ of GABA formation in $\mathrm{NaCl}$-treated soybeans, as noted in the paper by Xing et al. ${ }^{10}$ and our previous research, ${ }^{29}$ respectively. This difference may be due to different abiotic stresses and sampling positions. The mechanism of GABA in higher plants depends on the severity and duration of the stress and the growth conditions as well as the developmental stages of the plants. Furthermore, Glu is the direct substrate for GABA accumulation in the GABA shunt. Hence, in the present study, the Glu contents in both organs of aminoxyacetate-treated fava beans were increased compared with those of the control during germination, since the GAD activity was significantly inhibited.

\section{Conclusions}

Aminoxyacetate can be the most appropriate inhibitor of GAD activity in vivo, and it can be conveniently used to study the contribution ratio of GABA shunt and polyamine degradation pathway for GABA formation. It can be inferred that at least $43.9 \%$ and $81.5 \%$ of GABA accumulations were supplied by the GABA shunt in cotyledon and embryo, respectively, under hypoxia-NaCl stress.

\section{Conflicts of interest}

There are no conflicts of interest to declare.

\section{Acknowledgements}

This work was financially supported by the Natural Science Foundation of China (31501401), Natural Science Foundation of Jiangsu Province (BK 20150448) and Natural Science Foundation of Colleges and Universities in Jiangsu Province (15KJB550010) and Postgraduate Research \& Practice Innovation Program of Jiangsu Province (SJLX18_0616)

\section{References}

1 M. G. Erlander and A. J. Tobin, The structural and functional heterogeneity of glutamic acid decarboxylase: a review, Neurochem. Res., 1991, 16, 215-226. 
2 R. Yang, Q. Guo and Z. Gu, GABA shunt and polyamine degradation pathway on $\gamma$-aminobutyric acid accumulation in germinating fava bean (Vicia faba L.) under hypoxia, Food Chem., 2013, 136, 152-159.

3 Y. Yin, R. Yang, Q. Guo and Z. Gu, $\mathrm{NaCl}$ stress and supplemental $\mathrm{CaCl}_{2}$ regulating GABA metabolism pathways in germinating soybean, Eur. Food Res. Technol., 2014, 238, 781-788.

4 Y. Guo, R. Yang, H. Chen, Y. Song and Z. Gu, Accumulation of $\gamma$-aminobutyric acid in germinated soybean (Glycine $\max$ L.) in relation to glutamate decarboxylase and diamine oxidase activity induced by additives under hypoxia, Eur. Food Res. Technol., 2012, 234, 679-687.

5 X. Yan, P. Wang, T. Zhou, Z. Gu and R. Yang, NaCl-CaCl 2 treatment enhancing nutritional and functional quality of mung bean sprouts, Emir. J. Food Agric., 2017, 29, 123-131.

6 D. Del Rio, A. Rodriguez-Mateos, J. P. Spencer, M. Tognolini, G. Borges and A. Crozier, Dietary (poly) phenolics in human health: structures, bioavailability, and evidence of protective effects against chronic diseases, Antioxid. Redox Signaling, 2013, 18, 1818-1892.

7 E. García-Martín, G. Esguevillas, M. Serrador, H. AlonsoNavarro, F. Navacerrada, G. Amo, E. García-Albea, J. A. Agúndez and F. J. Jiménez-Jiménez, Gammaaminobutyric acid (GABA) receptors GABRA4, GABRE, and $G A B R Q$ gene polymorphisms and risk for migraine, $J$. Neural Transm., 2018, 4, 1-10.

$8 \mathrm{~N}$. Bouche and $\mathrm{H}$. Fromm, GABA in plants: just a metabolite?, Trends Plant Sci., 2004, 9, 110-115.

9 B. J. Shelp, G. G. Bozzo, C. P. Trobacher, A. Zarei, K. L. Deyman and C. J. Brikis, Hypothesis/review: Contribution of putrescine to 4-aminobutyrate (GABA) production in response to abiotic stress, Plant Sci., 2012, 193, 130-135.

10 S. G. Xing, Y. B. Jun, Z. W. Hau and L. Y. Liang, Higher accumulation of gamma-aminobutyric acid induced by salt stress through stimulating the activity of diarnine oxidases in Glycine $\max$ (L.) Merr. roots, Plant Physiol. Biochem., 2007, 45, 560-566.

11 R. Yang, H. Chen and Z. Gu, Factors influencing diamine oxidase activity and $\gamma$-aminobutyric acid content of fava bean (Vicia faba L.) during germination, J. Agric. Food Chem., 2011, 59, 11616-11620.

12 C. Dent, W. Stepka and F. Steward, Detection of the free amino acids of plant cells by partition chromatography, Nature, 1947, 160, 682-683.

13 Y. Guo, H. Chen, Y. Song and Z. Gu, Effects of soaking and aeration treatment on $\gamma$-aminobutyric acid accumulation in germinated soybean (Glycine max L.), Eur. Food Res. Technol., 2011, 232, 787-795.

14 R. Yang, Y. Yin, Q. Guo and Z. Gu, Purification, properties and cDNA cloning of glutamate decarboxylase in germinated faba bean (Vicia faba L.), Food Chem., 2013, 138, 1945-1951.

15 S. H. Oh, W. G. Choi, I. T. Lee and S. J. Yun, Cloning and characterization of a rice cDNA encoding glutamate decarboxylase, BMB Rep., 2005, 38, 595-601.
16 G. Baum, Y. Chen, T. Arazi, H. Takatsuji and H. Fromm, A plant glutamate decarboxylase containing a calmodulin binding domain. Cloning, sequence, and functional analysis, J. Biol. Chem., 1993, 268, 19610-19617.

17 P. V. Taberner, M. J. Pearce and J. C. Watkins, The inhibition of mouse brain glutamate decarboxylase by some structural analogues of L-glutamic acid, Biochem. Pharmacol., 1977, 26, 345-349.

18 R. Yang, Y. Yin, L. Guo and Z. Gu, Sequence analysis of diamine oxidase gene from fava bean and its expression related to $\gamma$-aminobutyric acid accumulation in seeds germinating under hypoxia-NaCl stress, J. Sci. Food Agric., 2014, 94, 1585-1591.

19 Q. Y. Bai, M. Q. Chai, Z. X. Gu, X. H. Cao, Y. Li and K. L. Liu, Effects of components in culture medium on glutamate decarboxylase activity and gamma-aminobutyric acid accumulation in foxtail millet (Setaria italica L.) during germination, Food Chem., 2009, 116, 152-157.

20 Y. Seher, O. Filiz and B. Melike, Gamma-amino butyric acid, glutamate dehydrogenase and glutamate decarboxylase levels in phylogenetically divergent plants, Plant Syst. Evol., 2013, 299, 403-412.

21 F. J. Turano and T. K. Fang, Characterization of two glutamate decarboxylase cDNA clones from Arabidopsis, Plant Physiol., 1998, 117, 1411-1421.

22 W. A. Snedden, T. Arazi, H. Fromm and B. J. Shelp, Calcium/ calmodulin activation of soybean glutamate decarboxylase, Plant Physiol., 1995, 108, 543-549.

23 M. Takayama, C. Matsukura, T. Ariizumi and H. Ezura, Activating glutamate decarboxylase activity by removing the autoinhibitory domain leads to hyper $\gamma$-aminobutyric acid (GABA) accumulation in tomato fruit, Plant Cell Rep., 2017, 36, 103-116.

24 F. Roberts, P. V. Taberner and R. G. Hill, The effect of 3mercaptopropionate, an inhibitor of glutamate decarboxylase, on the levels of GABA and other amino acids, and on presynaptic inhibition in the rat cuneate nucleus, Neuropharmacology, 1978, 17, 715-720.

25 T. Tsushida and T. Murai, Conversion of glutamic acid to $\gamma$ aminobutyric acid in tea leaves under anaerobic conditions, Agric. Biol. Chem., 1987, 51, 2865-2871.

26 R. Yang, Y. Yin, Q. Guo and Z. Gu, Purification, properties and cDNA cloning of glutamate decarboxylase in germinated faba bean (Vicia faba L.), Food Chem., 2013, 138, 1945-1951.

27 J. M. Barbosa, N. K. Singh, J. H. Cherry and R. D. Locy, Nitrate uptake and utilization is modulated by exogenous $\gamma$-aminobutyric acid in Arabidopsis thaliana seedlings, Plant Physiol. Biochem., 2010, 48, 443-450.

28 J. J. Molina-Rueda, M. B. Pascual, F. M. Cánovas and F. Gallardo, Characterization and developmental expression of a glutamate decarboxylase from maritime pine, Planta, 2010, 232, 1471-1483.

29 Y. Yin, R. Yang and Z. Gu, Organ-specific proteomic analysis of NaCl-stressed germinating soybeans, J. Agric. Food Chem., 2014, 62, 7233-7244. 